\title{
Serum Concentrations of Angiotensin, C-Reactive Protein, Interleukin-8, and Tumor Necrosis Factor- $\alpha$ in Train Driver Population
}

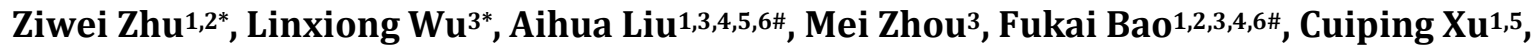 \\ Jiaru Yang1,5, Hua Zhao ${ }^{1,3,4}$ \\ ${ }^{1}$ Yunnan Key Laboratory for Tropical Infectious Diseases, Kunming, China \\ ${ }^{2}$ Department of Microbiology and Immunology, School of Basic Medical Science, Kunming Medical University, \\ Kunming, China \\ ${ }^{3}$ Yunnan Collaborative Innovation Center for Public Health and Disease Control, Kunming, China \\ ${ }^{4}$ The Institute for Tropical Medicine, Kunming Medical University, Kunming, China \\ ${ }^{5}$ Department of Biochemistry and Molecular Biology, School of Basic Medical Science, Kunming Medical \\ University, Kunming, China \\ ${ }^{6}$ Yunnan Province Base for International Scientific and Technological Cooperation in Tropical Diseases, Kunming, \\ China \\ Email: "Iunaliu123@126.com, "baofukai@126.com
}

Received 1 November 2015; accepted 1 March 2016; published 4 March 2016

Copyright (C) 2016 by authors and Scientific Research Publishing Inc.

This work is licensed under the Creative Commons Attribution International License (CC BY). http://creativecommons.org/licenses/by/4.0/

(c) (i) Open Access

\section{Abstract}

Train drivers are engaged in high-stress job. It may induce sleep, fatigue, and alertness loss at work, and endanger public safety. It's unclear that cytokines of train driver would be influenced by their job. The research considers the hypothesis that stressful professions, such as train driver, influence the body's immune system through the long-time and high-pressure working, and change production of neuro-immune factors. Using enzyme linked immunosorbent assay (ELISA), several neuro-immune factors were assayed among train drivers $(\mathrm{N}=82)$ and health blood donors $(\mathrm{N}=80)$ enrolled in the Yunnan Collaborative Innovation Center for Public Health and Disease Control. The concentrations of angiotensin, C-reactive protein (CRP), interleukin-8 (IL-8), and tumor necrosis factor-alpha (TNF- $\alpha$ ) were determined. Kruskal-Wallis test and Dunn's multiple comparisons test were performed for overall comparison between groups and for pairwise comparison, respectively. Statistical significance level was set at $P<0.05$. The profession of train driving

\footnotetext{
*These authors contribute equally to the paper.

"Corresponding authors.
}

How to cite this paper: Zhu, Z.W., Wu, L.X., Liu, A.H., Zhou, M., Bao, F.K., Xu, C.P., Yang, J.R. and Zhao, H. (2016) Serum Concentrations of Angiotensin, C-Reactive Protein, Interleukin-8, and Tumor Necrosis Factor- $\alpha$ in Train Driver Population. Open Journal of Immunology, 6, 7-13. http://dx.doi.org/10.4236/oji.2016.61002 
was not associated with significant increases or decreases in the systemic levels of inflammatory (CRP, IL-8, and TNF- $\alpha$ ), but it was associated with the high expression of angiotensin in vivo. These findings suggest that the job of train driving may not be associated with significant alterations in systemic immune condition, but arouse the level of angiotensin.

\title{
Keywords
}

\author{
Train Driver, Occupational Stress, Angiotensin, C-Reactive Protein, Interleukin-8, Tumor Necrosis \\ Factor-Alpha
}

\section{Introduction}

Train driving is classified as a high-level safety critical job. Safety critical workers are defined as those whose action or inaction, due to ill-health, may directly lead to a serious incident affecting the public. The health and fitness of these workers, especially their vigilance and attentiveness to their job are of paramount importance [1]. Sleepiness and fatigue are frequent problems in railway transportation with occasional monotony and irregular shift schedules [2]. The subjective workload of train drivers is relatively high. While attentional and information-processing requirements are increasing in many train driving tasks as a result of new technology, the driver's work is often subject to occasional periods of monotony [3]. The driver is often alone in the locomotive cabin. Driving is focused on controlling speed and acceleration but the driver also has to concentrate on the control of different dynamic forces, upcoming terrain, traffic signs and communication [4]. Those occupational stressors are recognized significant factors in influence of health condition of exposed workers [5].

The immune/inflammatory response occurs in response to infection, injury, and stress, where it helps to fight infection and repair damaged tissue, but can also occur for extended periods of time after infection, injury, and stress. Chronic, low-grade inflammation is now considered to be intimately linked to the development of diabetes and cardiovascular disease [6] [7]. Also, such "immune-stress" inflammation is increasingly implicated as a mediating factor relating stress to cardiovascular disease and is strongly linked to depression [8] [9]. However, its connection with train driving and, by extension, occupational stress is still uncertain. Answers to these questions will help to better understand stress condition of train driving, but also to develop new prophylactic strategies to care health of train drivers.

Angiotensin, the principal stress component of the renin-angiotensin aldosterone system (RAAS), plays an important role in the structural and functional regulation of the cardiovascular and renal system [10]. The RAAS pathway includes 3 important components of renin, angiotensin, and aldosterone. Angiotensin raises blood pressure by a number of actions, with the most important ones being vasoconstriction and sympathetic nervous stimulation. In addition to the regulation of blood pressure, angiotensin plays a major role in the sodium metabolism and renal hemodynamics [11] [12].

C-reactive protein (CRP) is a non-specific acute phase protein produced in the liver [13]. CRP, an important serum marker of inflammation, is used clinically as unspecific marker for inflammation [14]-[16]. In acute phase response, CRP levels can raise up to 1000-fold compared to normal within a few hours and be quite stable in the same individual across 24 hours [17] [18]. Furthermore, CRP is a strong predictor of cardiovascular diseases [19].

During the immune response, the cytokine interleukin-8 (IL-8, also called CXCL8 according to the chemokine nomenclature) functions as a strong chemoattractant for polymorphonuclear leukocytes helping to direct these cells to infected/injured sites. In addition to its potent chemotactic activity, it can induce proliferation and migration of cancer cells [20]. Because elevated IL-8 levels are associated with cardiovascular disease and negative psychological consequences, IL-8 is postulated to be a chronic stress biomarker [21].

Tumor necrosis factor-alpha (TNF- $\alpha$ ) is a pleiotropic cytokine known to play a major role in host defense mechanisms, initiating a beneficial local inflammation which in excess, however, may cause tissue damage [22] [23]. TNF- $\alpha$ is one inflammatory mediator that has been implicated in carcinogenesis, due to its participation in chronic inflammatory diseases [18] [24]. 


\section{Methods}

\subsection{Ethics}

The ethics committee of the Kunming Medical University approved the Yunnan Train Driver Risk Factor Surveillance and written informed consent was obtained from each participant before data and sample collection.

\subsection{Study Design and Measures}

This study was enrolled in the Yunnan collaborative Innovation center for public health and disease control. In this cross-sectional study we examined 82 train drivers employed by Kunming Railway Transportation Bureau of Kunming, China and 80 health blood donors occupied in different professions. An informed written consent was obtained from each participant. Medical and occupational history was collected from each subject.

From each participant, a whole blood sample was taken for tests after 12 hours of fasting, serum sample were collected and frozen at $-80^{\circ} \mathrm{C}$ for late use. The concentrations of angiotensin, C-reactive protein (CRP), interleukin-8 (IL-8), and tumor necrosis factor-alpha (TNF- $\alpha$ ) were measured in serum respectively using Human Elisa Kits to these factors (Huijia, Xiamen, China) by iMark microplate reader (Bio-Rad USA).

\subsection{Statistics}

Data were analyzed by GraphPad Prism 6 for Windows. Kruskal-Wallis test and Dunn's multiple comparisons test were performed for overall comparison among groups and for pairwise comparison, respectively. A $P$ value $<0.05$ was considered statistically significant.

\section{Results}

Train drivers were different to health blood donors with respect to most demographic characteristics, such as a $100 \%$ percentage of male and older age. The mean age in the 82 train drivers was 37 years old, 32 years old in the short working age group ( $\leq 18$ years), and 43 years old in the long working age group ( $>18$ years). Health blood donors represented most other occupational population. They had more balanced sex ratio (45\% of male and $55 \%$ of female) and younger mean age (27 years old; Table 1 ).

Engaged in train driving resulted in a $43.4 \%$ increase in serum angiotensin concentrations relative to health blood donors $(P<0.0001$ for train drivers vs. health blood donors). In contrast, there were no statistically significant differences in concentrations for CRP $(P=0.1876$ for train drivers vs. health blood donors), IL-8 ( $P=$ 0.2767 for train drivers vs. health blood donors), or TNF- $\alpha(P=0.6952$ for train drivers vs. health blood donors $)$ between driver group and control group. Median with interquartile range serum concentrations of the four cytokines according to working years are shown in Figures 1(a)-(d). The magnitude of increase in angiotensin levels was $>40 \%$ for the short and long working age categories with a $44.6 \%$ increase in objects worked for $\leq 18$ years $(P<0.0001)$ and a $41.9 \%$ increase for $>18$ years $(P<0.0001)$, compared to health blood donors (Figure 1(a)). There was no evidence for a difference in the angiotensin effect between train drivers in short and long levels of working age in analyses restricted to train drivers $(P>0.9999)$. Further, no significant differences were observed for CRP, IL-8 and TNF- $\alpha$ level in train drivers short and long levels of working age compared to health blood donors (Figures 1(b)-(d)).

\section{Discussion}

For study of risk factors of human health, Schrodinger's cat is a good metaphor. Every cat is threatened by deadly poison, but some cats die, while others are survived. Their destiny is determined by the probability of the

Table 1. Demographic characteristics of the study population from a cross-sectional molecular epidemiology study.

\begin{tabular}{|c|c|c|c|c|}
\hline \multirow{2}{*}{ Characteristics } & Health blood donors & Train drivers & Short working age & Long working age \\
\hline & $(\mathrm{n}, \%)$ & $(\mathrm{n}, \%)$ & $\leq 18$ years $^{\mathrm{a}}(\mathrm{n}, \%)$ & $>18$ years $^{\mathrm{a}}(\mathrm{n}, \%)$ \\
\hline Population number (n, \%) & $80(100)$ & $82(100)$ & 45 (54.88) & $37(45.12)$ \\
\hline Mean age (SD) & $27( \pm 8)$ & $37( \pm 7)$ & $32( \pm 4)$ & $43( \pm 4)$ \\
\hline
\end{tabular}

${ }^{\mathrm{a}}$ Based on the median working age among the inquired train drivers in the overall study population. 


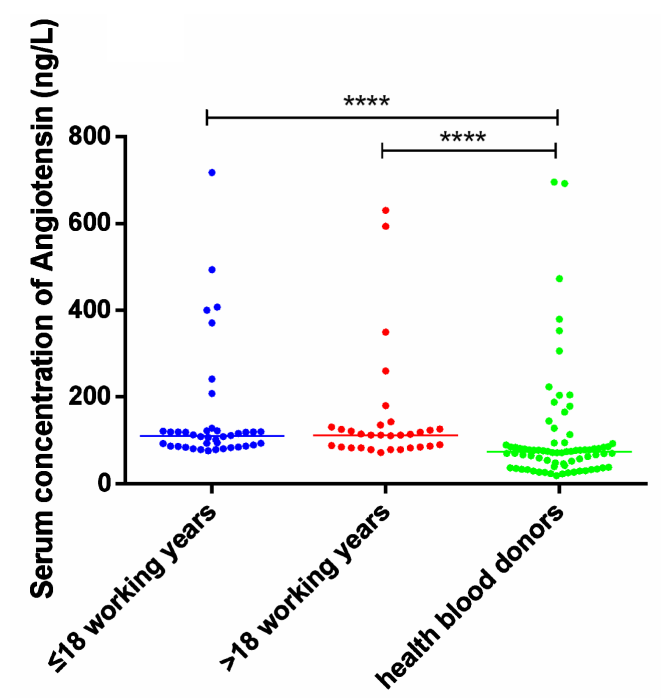

(a)

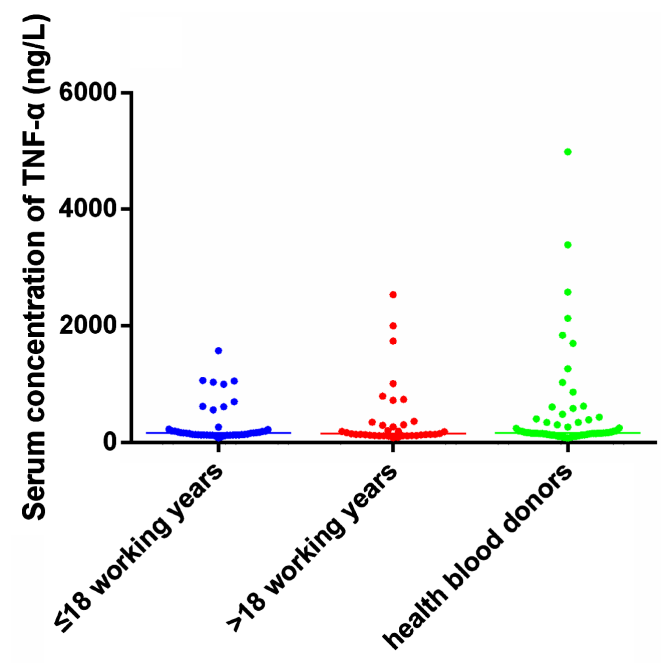

(c)

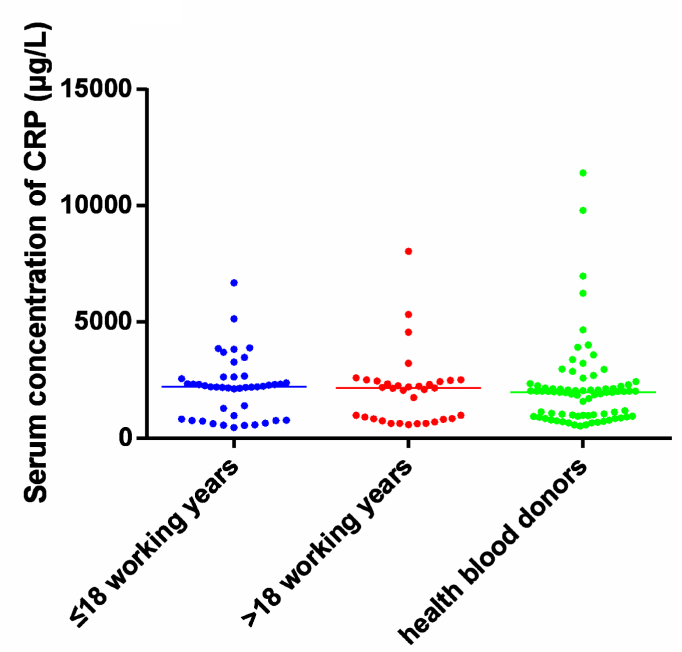

(b)

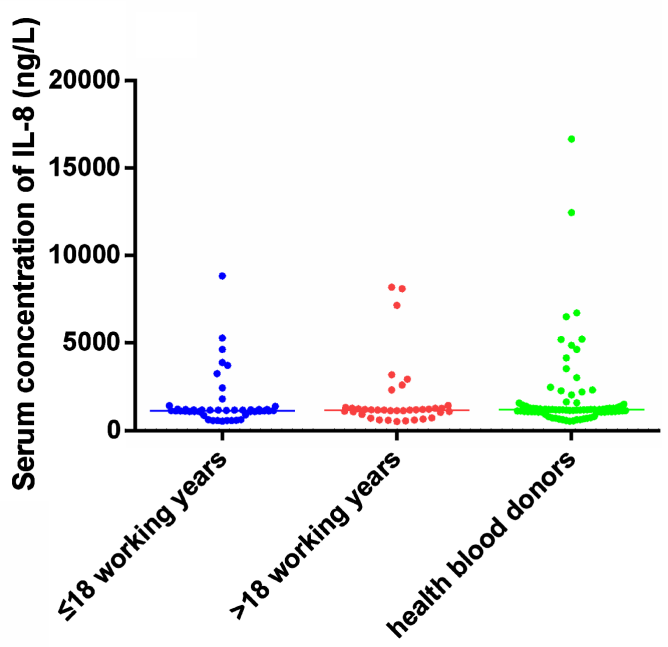

(d)

Figure 1. Serum concentrations of detected molecules in different group. (a)-(d) Dots and lines represent serum concentrations of detected molecules and median values in samples according to working years $(\leq 18$ years, $>18$ years, health blood donors). Eighteen years was the median working age of the inquired train drivers. $P$-values compare short working age group and long working age group to health blood donors, $P<0.05$ is indicated as significant difference.

being triggered poison. Just like the case, every train driver' destiny is like the cat's, which is facing many kinds of dangers, and what is different is the size of probability. The poison is the real danger that train drivers may face in their work, such as train out of control, operational errors, and so on. And work pressure, biochemical indicators in our research are like parts of poison triggering device. Our goal is to understand the process of the triggering and how to intervene. This study is the first step, namely to observe what is happened and what changes produced in the train driver's body.

To the best of our knowledge, this is the first study to consider the effect of working age on serum levels of angiotensin, CRP, IL-8 and TNF- $\alpha$ in adult train drivers. Our finding of angiotensin increase in train drivers, which was independent of other demographic factors that may influence serum cytokine levels, such as smoking status and recent infection, provides further indication that train driving can alter immune condition at relatively short working age.

Occupational stress is assumed to be a connection between immunological inflammatory functions and human 
diseases. Several lines of evidence have emerged suggesting that occupational stress can affect individual susceptibility for diseases such as diabetes, hypertension, coronary heart disease, metabolic syndrome, and dementia [9]. Indeed, the alteration of the immune parameters was observed among some specific occupational groups prone to workplace stress such as blue-collar workers, paratroops, and nurses [25]. Furthermore, work-related exhaustion can lead to various physical and psychological symptoms, and also may be associated with delayed decision-making, causing mistakes and heavy losses [21].

Although few studies evaluating changes in cytokine levels in relation to occupational stress have been conducted in train drivers, some studies in other jobs have indicated job stress is assumed to act as a potential risk factor in cellular or humoral immune responses and induce immunological imbalance by itself. One exploratory longitudinal analysis found that nurses who experienced high levels of stress presented with lower level of TNF- $\alpha$ [9]. However, a investigation in paratroops after parachuting observed significantly higher levels of serum hormones such as growth hormone, angiotensin, and TNF-a in the parachuting group [25] an analogous immune response was observed in the one previous occupational study, which found that the inflammatory marker (CRP) was raised in unemployed participants compared to working counterparts [8]. In addition, it is reported that urinary IL-8 is a convenient and non invasive strong biomarker of stress, both acute and over time. Again within the limited results of correlations, emergency physicians were observed that sleep deprivation in their night shifts also exacerbates CRP secretion [21]. Our results showing an increase in angiotensin levels in train drivers are consistent with this type of immune response, given that angiotensin is involved in the the kidney to hypertensive stimuli and the induction of hypertension [26]. Meanwhile, our results didn't find statistically significant increase for CRP, IL-8 and TNF- $\alpha$ in train drivers.

\section{Conclusion}

In summary, we found that the serum concentration of angiotensin was markedly increased in train drivers compared to health blood donors, and that this increase occurred in train drivers worked $\leq 18$ years as well as $>18$ years. Conversely, no change in CRP, IL-8 and TNF- $\alpha$ level related to train driving was evident. Given that cytokines alterations are suspected to play a role in work-related stress, and angiotensin plays an important role in stress processes, our findings provide additional evidence that angiotensin is increased in train drivers and may serve as useful biomarkers to assess possible abnormal stress in train drivers and to evaluate the health condition. However, our findings require replication in larger sample studies and in other occupational stress populations.

\section{Acknowledgements}

This work was supported by National Natural Science Foundation of China Grants (No. 81060134, 81371835, 31560051, 81560596); Natural Foundation of Yunnan Province grants (2012FB011, 2013FZ057, 2014FA011, 2014FB001).

\section{Competing Interest}

The authors have no competing interests.

\section{Authors' Contribution}

Fukai Bao was responsible for the integrity of the work as a whole. ZZ contributed to data analysis and interpretation and wrote the first draft. LW, MZ and AL contributed to the study design, data acquisition and analysis. $\mathrm{CX}, \mathrm{JY}$ and HZ contributed to data analysis and interpretation. All authors contributed to data interpretation and to manuscript drafting and approval.

\section{References}

[1] Loukzadeh, Z., Zare, Z., Mehrparvar, A.H., Mirmohammadi, S.J. and Mostaghaci, M. (2013) Fitness-for-Work Assessment of Train Drivers of Yazd Railway, Central Iran. International Journal of Occupational and Environmental Medicine, 4, 157-163.

[2] Harma, M., Sallinen, M., Ranta, R., Mutanen, P. and Müller, K. (2002) The Effect of an Irregular Shift System on Sleepiness at Work in Train Drivers and Railway Traffic Controllers. Journal of Sleep Research, 11, 141-151. 
http://dx.doi.org/10.1046/j.1365-2869.2002.00294.x

[3] Sallinen, M., Härmä, M., Mutanen, P., Ranta, R., Virkkala, J. and Müller, K. (2003) Sleep-Wake Rhythm in an Irregular Shift System. Journal of Sleep Research, 12, 103-112. http://dx.doi.org/10.1046/j.1365-2869.2003.00346.X

[4] Kim, S.E., Kim, H.R., Park, J.I., Lee, H.W., Lee, J., Byun, J. and Yim, H.W. (2014) The Association between Psychiatric Disorders and Work-Related Problems among Subway Drivers in Korea. Annals of Occupational and Environmental Medicine, 26, 39. http://dx.doi.org/10.1186/s40557-014-0039-7

[5] Jovanović, J. and Jovanović, M. (2004) Occupational Stress and Arterial Hypertension. Medicinski Pregled, 57, 153158. http://dx.doi.org/10.2298/MPNS0404153J

[6] Buyken, A.E., Goletzke, J., Joslowski, G., Felbick, A., Cheng, G., Herder, C. and Brand-Miller, J.C. (2014) Association between Carbohydrate Quality and Inflammatory Markers: Systematic Review of Observational and Interventional Studies. American Journal of Clinical Nutrition, 99, 813-833. http://dx.doi.org/10.3945/ajcn.113.074252

[7] Martynowicz, H., Janus, A., Nowacki, D. and Mazur, G. (2014) The Role of Chemokines in Hypertension. Advances in Clinical and Experimental Medicine, 23, 319-325. http://dx.doi.org/10.17219/acem/37123

[8] Hughes, A., McMunn, A., Bartley, M. and Kumari, M. (2015) Elevated Inflammatory Biomarkers during Unemployment: Modification by Age and Country in the UK. Journal of Epidemiology and Community Health, 69, 673-679. http://dx.doi.org/10.1136/jech-2014-204404

[9] Yoon, H.S., Lee, K.M. and Kang, D. (2014) Intercorrelation between Immunological Biomarkers and Job Stress Indicators among Female Nurses: A 9-Month Longitudinal Study. Frontiers in Public Health, 2, 157. http://dx.doi.org/10.3389/fpubh.2014.00157

[10] Opie, L.H. (2014) Cognitive Decline: Mechanisms and Proposed Role of the Renin-Angiotensin-Aldosterone System. Cardiovascular Journal of Africa, 25, 149-150.

[11] Ramanathan, G., Elumalai, R., Periyasamy, S. and Lakkakula, B. (2014) Role of Renin-Angiotensin-Aldosterone System Gene Polymorphisms and Hypertension-Induced End-Stage Renal Disease in Autosomal Dominant Polycystic Kidney Disease. Iranian Journal of Kidney Diseases, 8, 265-277.

[12] Balakumar, P. and Jagadeesh, G. (2014) Structural Determinants for Binding, Activation, and Functional Selectivity of the Angiotensin AT1 Receptor. Journal of Molecular Endocrinology, 53, R71-R92. http://dx.doi.org/10.1530/JME-14-0125

[13] Wong, J.Y., de Vivo, I., Lin, X., Fang, S.C. and Christiani, D.C. (2014) The Relationship between Inflammatory Biomarkers and Telomere Length in an Occupational Prospective Cohort Study. PLoS ONE, 9, e87348. http://dx.doi.org/10.1371/journal.pone.0087348

[14] Strang, F. and Schunkert, H. (2014) C-Reactive Protein and Coronary Heart Disease: All Said—Is Not It? Mediators of Inflammation, 2014, Article ID: 757123. http://dx.doi.org/10.1155/2014/757123

[15] de Ferranti, S.D. and Rifai, N. (2007) C-Reactive Protein: A Nontraditional Serum Marker of Cardiovascular Risk. Cardiovascular Pathology, 16, 14-21. http://dx.doi.org/10.1016/j.carpath.2006.04.006

[16] Emerging Risk Factors Collaboration, Kaptoge, S., Di Angelantonio, E., Pennells, L., Wood, A.M., White, I.R., Gao, P., Walker, M., Thompson, A., Sarwar, N., Caslake, M., Butterworth, A.S., Amouyel, P., Assmann, G. and Bakker, S.J. (2012) C-Reactive Protein, Fibrinogen, and Cardiovascular Disease Prediction. The New England Journal of Medicine, 367, 1310-1320. http://dx.doi.org/10.1056/NEJMoa1107477

[17] Zimmermann, O., Li, K., Zaczkiewicz, M., Graf, M., Liu, Z. and Torzewski, J. (2014) C-Reactive Protein in Human Atherogenesis: Facts and Fiction. Mediators of Inflammation, 2014, Article ID: 561428. http://dx.doi.org/10.1155/2014/561428

[18] Nadeem, R., Molnar, J., Madbouly, E.M., Nida, M., Aggarwal, S., Sajid, H., Naseem, J. and Loomba, R. (2013) Serum Inflammatory Markers in Obstructive Sleep Apnea: A Meta-Analysis. Journal of Clinical Sleep Medicine, 9, 10031012. http://dx.doi.org/10.5664/jcsm.3070

[19] Agrawal, A., Gang, T.B. and Rusinol, A.E. (2014) Recognition Functions of Pentameric C-Reactive Protein in Cardiovascular Disease. Mediators of Inflammation, 2014, Article ID: 319215. http://dx.doi.org/10.1155/2014/319215

[20] Pichert, A., Schlorke, D., Franz, S. and Arnhold, J. (2012) Functional Aspects of the Interaction between Interleukin-8 and Sulfated Glycosaminoglycans. Biomatter, 2, 142-148. http://dx.doi.org/10.4161/biom.21316

[21] Dutheil, F., Trousselard, M., Perrier, C., Lac, G., Chamoux, A., Duclos, M., Naughton, G., Mnatzaganian, G. and Schmidt, J. (2013) Urinary Interleukin-8 Is a Biomarker of Stress in Emergency Physicians, Especially with Advancing Age-The JOBSTRESS* Randomized Trial. PLoS ONE, 8, e71658. http://dx.doi.org/10.1371/journal.pone.0071658

[22] Landskron, G., De la Fuente, M., Thuwajit, P., Thuwajit, C. and Hermoso, M.A. (2014) Chronic Inflammation and Cytokines in the Tumor Microenvironment. Journal of Immunology Research, 2014, Article ID: 149185. http://dx.doi.org/10.1155/2014/149185 
[23] Jarrot, P.A. and Kaplanski, G. (2014) Anti-TNF-Alpha Therapy and Systemic Vasculitis. Mediators of Inflammation, 2014, Article ID: 493593. http://dx.doi.org/10.1155/2014/493593

[24] Nanau, R.M. and Neuman, M.G. (2014) Safety of Anti-Tumor Necrosis Factor Therapies in Arthritis Patients. Journal of Pharmaceutical Sciences, 17, 324-361.

[25] Wu, T.C., Xiong, Y.L., Chen, S., Leng, S.T., Hai, T. and Tanguay, R.M. (1999) Biochemical Changes of Plasma in Paratroops after Parachuting: A Preliminary Investigation. Space Medicine \& Medical Engineering, 12, $235-239$.

[26] Giani, J.F., Janjulia, T., Taylor, B., Bernstein, E.A., Shah, K., Shen, X.Z., McDonough, A.A., Bernstein, K.E. and Gonzalez-Villalobos, R.A. (2014) Renal Generation of Angiotensin II and the Pathogenesis of Hypertension. Current Hypertension Reports, 16, 477. http://dx.doi.org/10.1007/s11906-014-0477-1 